\title{
Giant Planets Can Act as Stabilizing Agents on Debris Disks
}

\author{
M. A. Muñoz-Gutiérrez ${ }^{1}$, B. Pichardo, and A. Peimbert \\ Instituto de Astronomía, Universidad Nacional Autónoma de México, Apdo. postal 70-264 \\ Ciudad Universitaria, México; mmunoz.astro@gmail.com \\ Received 2017 March 13; revised 2017 April 28; accepted 2017 May 18; published 2017 June 21
}

\begin{abstract}
We have explored the evolution of a cold debris disk under the gravitational influence of dwarf-planet-sized objects (DPs), both in the presence and absence of an interior giant planet. Through detailed long-term numerical simulations, we demonstrate that when the giant planet is not present, DPs can stir the eccentricities and inclinations of disk particles, in linear proportion to the total mass of the DPs; on the other hand, when the giant planet is included in the simulations, the stirring is approximately proportional to the mass squared. This creates two regimes: below a disk mass threshold (defined by the total mass of DPs), the giant planet acts as a stabilizing agent of the orbits of cometary nuclei, diminishing the effect of the scatterers; above the threshold, the giant contributes to the dispersion of the particles.
\end{abstract}

Key words: methods: numerical - planet-disk interactions - protoplanetary disks

\section{Introduction}

Apart from planets, our solar system comprises an enormous set of smaller objects that go from about $2000 \mathrm{~km}$ (dwarf planets) to about $10^{-6} \mathrm{~cm}$ (interplanetary dust). Here, we will refer to as a "debris disk" everything within a stellar system except for the host star and planets; we also mean for the system to be somewhat evolved and no longer forming planets (i.e., not a protoplanetary disk). As such, not much gas is expected to remain (Kral et al. 2017). In the case of the solar system, this is mainly the asteroid and Kuiper belts. When referring to extrasolar debris disks, authors usually focus on the dust component (easier to observe), instead of the larger (but less numerous) dwarf planets. However, it has also been assumed that there should be a spectrum of masses from dust to planetesimals that simultaneously coexist (e.g., Wyatt 2008 and references therein), as well as an effective mechanism to continuously produce dust in these systems (Backman \& Paresce 1993; Kenyon \& Bromley 2004).

The origin of debris disks is still uncertain, but it has been suggested that they might be the remnants of protoplanetary disks, which seem almost ubiquitous around newly formed stars (Haisch et al. 2001). The masses of protoplanetary disks are larger than $1 M_{\oplus}$ while debris disks are less massive than $1 M_{\oplus}$; it is believed that the clearing processes of planetary disks (e.g., accretion to the central star and planets, photoevaporation, chaotic dispersion) are not totally effective, leaving residual solid material behind that may be the origin of debris disks (Wyatt 2008).

The dust in debris disks could either be generated as part of the original dust component of the protoplanetary disk that was not cleared up (a.k.a. primordial dust), or, assuming the clearing processes also left behind large planetesimals $(\gtrsim 1 \mathrm{~km})$, produced by planetesimal collisions; the timescales observed for the older debris disks supports this latter scenario (Wyatt \& Dent 2002), although for younger ones, it might be directly the dust left behind by protoplanetary disks.

\footnotetext{
${ }^{1}$ Current address: UNESP_São Paulo State University, Grupo de Dinâmica Orbital e Planetologia, Av. Ariberto Pereira da Cunha, 333, Guaratinguetá, SP, 12516-410, Brazil.
}

Debris disks also seem to be common around young objects, and their presence sometimes lasts as long as the planetary system exists. The evolution of debris disks may be influenced by planet formation processes, which last for about $1 \mathrm{Gyr}$, the time required by the system to settle into a stable configuration (Wyatt 2008).

The first extrasolar debris disk was discovered from the thermal emission of dust observed with the IRAS satellite in the star Vega (Aumann et al. 1984). Since that time, about 1750 debris disks have been found by their dust emission (e.g., Oudmaijer et al. 1992; Mannings \& Barlow 1998; Cotten \& Song 2016), and we also expect a large quantity of dust-poor debris disk systems, similar to the Kuiper Belt (Moro-Martín et al. 2008; Wyatt 2008; Nesvorný et al. 2010; Vitense et al. 2012).

The morphological and dynamical characteristics of debris disks are influenced by the configuration of the hosting planetary system (e.g., Mouillet et al. 1997; Wyatt et al. 1999; Matthews et al. 2014; Nesvold \& Kuchner 2015; Lee \& Chiang 2016; Nesvold et al. 2016, 2017); in particular, the formation of giant planets has an important dynamical effect on protoplanetary disks. Once one or more planets have formed and the gas has dissipated, the planets will continue to gravitationally influence the debris disk. There are important effects from the presence of a planet close to a debris disk, such as stirring through secular and resonant perturbations and resonant gaps (Wyatt 2008; Mustill \& Wyatt 2009), among others. A nearby example is the case of the Kuiper Belt, which shows evidence of sculpting due to the migration of Neptune (Chiang et al. 2007; Morbidelli et al. 2008; Nesvorný 2015); all these works consider the giant planet as an orbital "disorderer" agent. In this work, we are quantifying an effect presented in Muñoz-Gutiérrez et al. (2015); specifically we demonstrate that giant planets, depending on the total mass of a given debris disk, can also act as a stabilizing mechanism on the cometary nuclei, and it can quench the dynamical evaporation induced by the presence of dwarf-planet-sized objects (DPs) on debris disks.

This paper is organized as follows. In Section 2, we provide the details of the numerical simulations we performed; we also describe the distributions of DPs, and the debris disk and the 
giant planet used in this work. In Section 3, we present and discuss the results of our simulations, while in Section 4, we discuss and enumerate the main conclusions of this work.

\section{Simulations}

We perform a large set of simulations of debris disks composed of 50 DPs and 5000 massless test particles which represent cometary nuclei. All of our simulations were performed using the mixed symplectic integrator of the MERCURY package (Chambers 1999) with an initial time step of 180 days and an accuracy parameter for the BulirshStöer integrator of $10^{-10}$, which solves the evolution of test particles when their distance to any major body is smaller than 3 Hill radii $\left(R_{\mathrm{H}}=a_{p}\left(M_{p} / 3 M_{\odot}\right)^{1 / 3}\right.$; where $a_{p}$ and $M_{p}$ are the semimajor axis of the orbit and the mass of the planet, respectively). Most of our simulations are $1 \mathrm{Gyr}$ long, but for some cases we extended the simulations to $2 \mathrm{Gyr}$.

\subsection{Test Particles's Initial Conditions: Random Cold Debris Disk}

Taking as a reference our own solar system (or more precisely, the Kuiper Belt), we generate a random belt of debris distributed between 38 and 50 au in semimajor axis, $a$, which consists of 5000 test particles. The direction of the angular momentum is determined as a two-dimensional Gaussian distribution centered on the $Z$-axis with a width of 1.2 in each of the $X$ and $Y$ axes (see the description in Muñoz-Gutiérrez et al. 2015). This translates to an average inclination of $\mu_{i}=1.47$, an inclination dispersion of $\sigma_{i}=0.75$, and at the same time defines a homogeneous random distribution of the longitude of the ascending node, $\Omega$, between $0^{\circ}$ and $360^{\circ}$. The particle eccentricity, $e$, and the argument of pericenter, $\omega$, are defined similarly: first by randomly assigning the locus of the second focus of each elliptical orbit (the first being the Sun) with a Gaussian probability distribution in the $X Y$ plane, with a center coinciding with the Sun and a width of 0.03 along each axis; this translates to an average eccentricity, $\mu_{e}=0.037$, an eccentricity dispersion, $\sigma_{e}=0.02$, and at the same time defines a homogeneous random distribution of $\omega$ between $0^{\circ}$ and $360^{\circ}$. Finally, the mean anomaly, $M$, is randomly generated between $0^{\circ}$ and $360^{\circ}$.

This disk, with the same random seeds, is used in all our simulations as the test-particle/cometary-nuclei initial conditions.

\subsection{Dwarf Planet Distributions}

Our simulations require a suitable number of scatterers embedded in our cold belt in order to be both (a) perturbative enough in the presence of a giant planet and (b) computationally fast enough to run the required number of simulations in a feasible amount of time. From the experiments performed in Muñoz-Gutiérrez et al. (2015), we consider that 50 scatterers are adequate to fulfill both requirements. The 50 DPs we use represent the 50 largest DPs in such a debris disk. In the rest of this work, we use different distributions of 50 DPs each, which differ from each other by the maximum inclination of the set, the total mass of the DPs, and the index of their differential mass distribution, $\alpha$. Below we describe the different DP distributions employed.
First, we used a differential mass distribution (dMD) derived from the differential size distribution (dSD). A dSD is given by

$$
\frac{d N}{d D} \propto D^{-\alpha^{\prime}},
$$

where $N$ is the number of objects larger than $D$. It has been shown that $\alpha^{\prime}$ values range from 3.5 for belts in collisional equilibrium (Dohnanyi 1969), to 4.0 or 4.5 for the largest objects in the Kuiper Belt (Fraser \& Kavelaars 2008; Fraser et al. 2008). It is straightforward to obtain a dMD by considering that for a constant density $D \propto M^{1 / 3}$; thus, a $\mathrm{dMD}$ will be given by

$$
\frac{d N}{d M} \propto M^{-\alpha},
$$

where $N(M)$ is the number of objects more massive than $M$ and where $\alpha=\left(\alpha^{\prime}+1\right) / 3-1$ takes values from $\sim 1.8$ for disks in collisional equilibrium, to $\sim 2$ or $\sim 2.2$ for the largest Kuiper Belt objects.

In order to compute a random mass distribution of DPs that follows Equation (2), for each $\alpha$ we assigned the mass of the $n$th planet to be

$$
m_{n}=\left(\frac{K_{\alpha}}{(\alpha-1) n^{\prime}}\right)^{1 /(1-\alpha)} .
$$

Here, $n^{\prime}$ is a random real number in the interval $[0.5,50]$, while $K_{\alpha}$ is a constant related to the largest mass allowed in the distribution of DPs. We set the maximum value of $n^{\prime}$ to 50 to represent the 50 heaviest planets of our sample; in a more realistic simulation, we would expect to have 50 more objects with $50<n^{\prime}<100$ (and even a greater number of smaller objects); however, these objects are expected to have a much smaller contribution to the dynamics of the disk. We set the minimum value of $n^{\prime}$ to 0.5 to avoid the possibility of a single very large planet completely dominating the dynamics of the disk; $K_{\alpha}$ is set so that the total mass of the 50 DPs equals $(\sqrt{5})^{q} \times M_{\mathrm{CKB}}$, where $M_{\mathrm{CKB}}$ is the estimated mass of the classical Kuiper Belt, set as $M_{\mathrm{CKB}}=0.01 M_{\oplus}$ (Bernstein et al. 2004; Fraser et al. 2014), while $q$ is an integer in the interval $[0,4]$. This covers the cases where the addition of the masses of the 50 DPs equal $0.01,0.0223,0.05,0.1118$, and $0.25 M_{\oplus}$. Therefore, $K_{\alpha}$ varies for each index $\alpha$ considered and for the total mass of DPs. For example, for an index $\alpha=2$ and a total mass of $0.01 M_{\oplus}, K_{\alpha}$ will be of the order of the mass of Pluto, while the heaviest possible planet in this distribution would have twice Pluto's mass. It should be noted that in our simulations, the total mass of the debris disk corresponds to the sum of the masses of the 50 DPs.

As an example, we show in Figure 1 the masses and positions, in semimajor axis, of three distributions of DPs; they differ in the index of their dMD but their total mass equals $0.01 M_{\oplus}$ in all cases. This distribution is general throughout this work in the sense that the position in $a$ will be the same, since the random seed we use to define the DPs is the same for all simulations. Note, however, that the individual mass of each DP will be rescaled; although the sets of $m_{n}$ will not be identical, the sets of $n^{\prime}$ will be identical, which will, using Equation (3), produce the sets of $m_{n}$ (for the $\alpha$ and $K_{\alpha}$ that correspond to each simulation). The particular $a$ distribution 


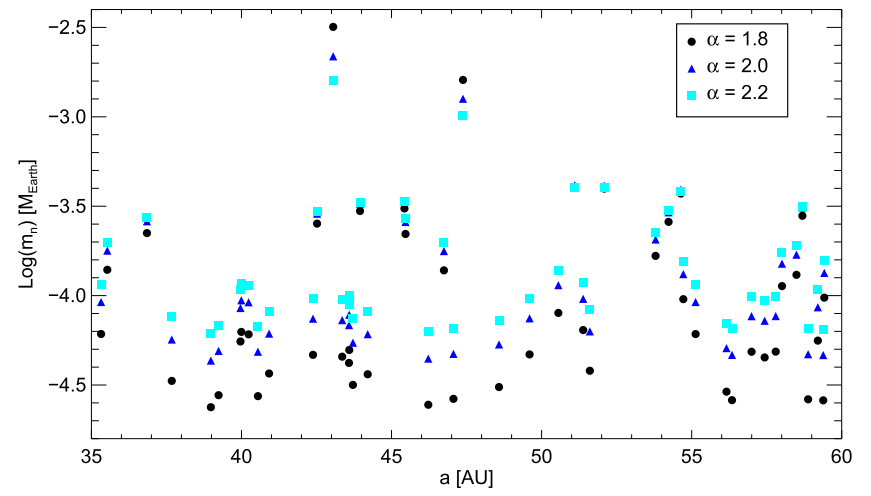

Figure 1. Distribution of DPs for different dMD indexes. The mass for each $\mathrm{DP}$ in the distribution is plotted against its semimajor axis, for three different distributions of DPs with total mass $0.01 M_{\oplus}$ but with different $\alpha$ indexes, as indicated in the figure.

generated in this work has the advantage that the two heaviest DPs lie almost in the middle of the debris disk, thus their effect is highlighted, while the smallest DPs are randomly exerting their influence along the disk and beyond its limits.

Regarding the maximum possible inclination of DPs, $i_{\max }$, we explore three different cases: cold distributions have $i_{\max }=5^{\circ}$, warm distributions have $i_{\max }=15^{\circ}$, and hot distributions have $i_{\max }=30^{\circ}$. For each case, inclinations of DPs are assigned randomly between 0 and $i_{\max }$.

The remaining orbital parameters are the same for all distributions as follows: $a$ is randomly determined from a uniform distribution between 35 and $60 \mathrm{au}, e$ is randomly distributed between 0 and 0.1 , and finally $\omega, \Omega$, and $M$ are set randomly between $0^{\circ}$ and $360^{\circ}$.

\subsection{Giant Neptune-like Planet}

The influence of DP distributions on the debris disk is tested with and without the presence of an interior giant planet; such a giant shares all the orbital and physical characteristics of Neptune, except for its nominal inclination. The kinematics of the outer solar system are dominated by Neptune-in particular, any debris disk should align itself with Neptune's inclination. The same will happen with any other debris disk with an interior giant planet. To avoid an initial rearrangement of test particles, we set the giant inclination to $0^{\circ}$, instead of 1.8 as defined in the solar system.

Table 1 shows the physical parameters of the Neptune-like planet used in this work, as well as its initial orbital osculating parameters for the simulations. A central star of $1 M_{\odot}$ is present in all simulations.

Table 2 shows a summary of all parameters we can vary in each simulation. There are 90 different scenarios resulting from combinations of these parameters, but we have not run all possible cases. In what follows, when needed, we will refer to a particular simulation with the generic name $\left|G P_{(Y / N)} / \alpha x / i_{\max } y / M_{\mathrm{DP}} z\right|$, where $G P_{(Y / N)}$ refers to the inclusion or not of the giant planet in the simulation, and $(x, y, z)$ are the possible values of each varied parameter. For example, a simulation including the giant planet, with a distribution of DPs with a dMD index $\alpha=2$, a maximum inclination $i_{\max }=5^{\circ}$, and a total mass of $0.05 M_{\oplus}$, will be labeled $\left|G P_{Y} / \alpha 2 / i_{\max } 5 / M_{\mathrm{DP}} 5\right|$.
Table 1

Giant Planet's Physical and Initial Orbital Parameters

\begin{tabular}{lc}
\hline \hline Constant & Value \\
\hline $\mathrm{GM}\left(\mathrm{km}^{3} \mathrm{~s}^{-2}\right)$ & $6.836534062 \times 10^{6}$ \\
Radius $(\mathrm{km})$ & 24043 \\
$a(\mathrm{au})$ & 30.09598340353801 \\
$e$ & 0.01078167440459 \\
$i\left(^{\circ}\right)$ & 0.0 \\
$\omega\left(^{\circ}\right)$ & 272.2034594991346 \\
$\Omega\left({ }^{\circ}\right)$ & 131.7818550161205 \\
$M\left({ }^{\circ}\right)$ & 288.9806490025501 \\
\hline
\end{tabular}

Table 2

Summary of Varied Parameters in Different Simulations

\begin{tabular}{lccccc}
\hline \hline Parameter & Value & & & & \\
\hline GP & Yes & No & & & \\
$\alpha$ & 1.8 & 2.0 & 2.2 & & \\
$i_{\max }$ & $5^{\circ}$ & $15^{\circ}$ & $30^{\circ}$ & & 21.18 \\
$M_{\text {DPs }}$ & 1.0 & 2.23 & 5.0 & 25.0 \\
\hline
\end{tabular}

Note. Here, $M_{\mathrm{DPs}}$ is given in units of masses of the classical Kuiper Belt, $M_{\mathrm{CKB}}=0.01 M_{\oplus}$.

\section{Results}

DPs have been commonly underestimated when considering the dynamical evolution of debris disks. Those massive bodies can be considered as the far end of the size distribution of protoplanetary and debris disks. In this work, we show the importance of DPs in the secular evolution of a cold debris disk; we do this as a function of the total mass of DPs and other critical orbital characteristics.

To analyze the changes in the global properties of our debris disk, we have measured the evolution of the average orbital elements, $\langle e\rangle$ and $\langle i\rangle$, as well as their associated standard deviations, $\sigma_{e}$ and $\sigma_{i}$, for the whole population of the disk. We have not shown the evolution of $\langle a\rangle$ because, in general, this parameter does not evolve significantly; thus, it cannot provide us with valuable information. Of course, some particles will experience a significant change in semimajor axis due to perturbations by the DPs, but on average, the position of the disk remains unchanged.

On the other hand, both $\langle e\rangle$ and $\langle i\rangle$ are related to the dynamical heating and the thickness of the disk; this is because of the maximal excursions of particles above the reference plane, related to $\langle i\rangle$, and in the radial direction, related to $\langle e\rangle$.

\subsection{Effect of the Heavy End of the Mass Distributions}

As we mentioned earlier in Section 2.2, we have tested three possibilities for the dMD index of DPs, where the lower limit, $\alpha=1.8$, relates to the heavy end of the size distribution of a debris disk in collisional equilibrium, while the upper limit, $\alpha=2.2$, relates to the sizes of the largest objects in the Kuiper Belt, which constitutes our reference system.

Figure 2 shows the evolution of $\langle e\rangle$ (left upper panel), $\langle i\rangle$ (left bottom panel), $\sigma_{e}$ (right upper panel), and $\sigma_{i}$ (right bottom panel) for six simulations that share two parameters: $i_{\max }=5^{\circ}$ (a cold population of DPs) and their total mass, $M_{\mathrm{DPs}}=0.01 M_{\oplus}$. Three simulations included the giant planet (shown in thin lines), while the other three did not (shown in thick lines); we used three 

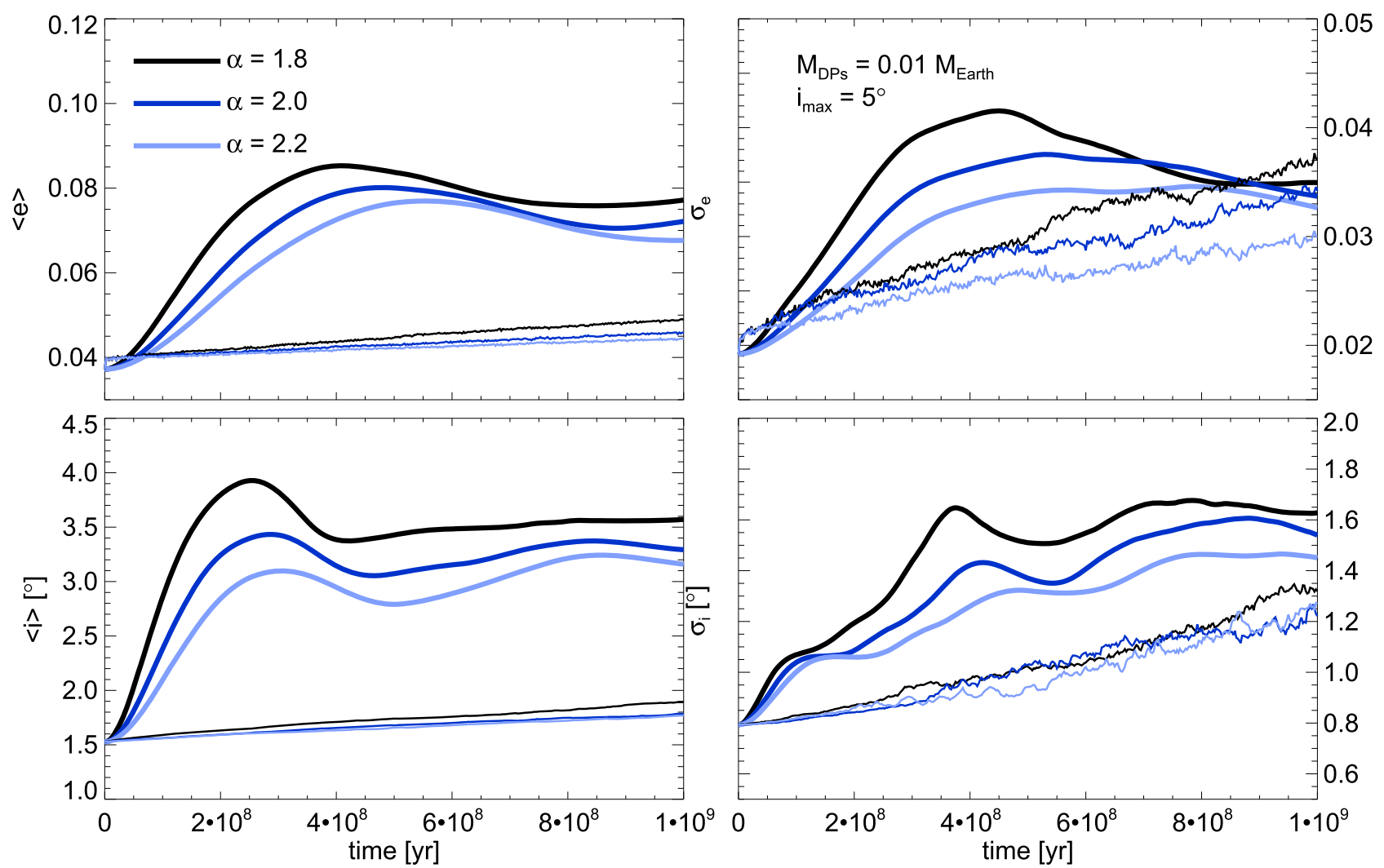

Figure 2. Evolution of the $\langle e\rangle, \sigma_{e},\langle i\rangle$, and $\sigma_{i}$ of the particles of debris disks with $M_{\mathrm{DPs}}=0.01 M_{\oplus}$ and $i_{\max }=5^{\circ}$. Black lines represent disks with $\alpha=1.8$, dark blue lines disks with $\alpha=2.0$, and light blue lines disks with $\alpha=2.2$; thick lines are for simulations without the giant planet, while thin lines are simulations that included the Neptune-like giant.

different indexes $\alpha$ in each case: black lines are simulations in which the DP distribution follows a dMD with $\alpha=1.8$, blue lines for $\alpha=2.0$, and light blue lines for $\alpha=2.2$.

Evident from Figure 2 is the weak dependence of the global evolution of the disk on the parameter $\alpha$ used to generate the distribution of DPs. Nonetheless, it is worth noting that for simulations without the giant planet, the $\alpha=1.8$ distribution (thick black lines) leads to a higher global stirring of the orbits than the $\alpha=2.2$ case (thick light blue lines), while the $\alpha=2.0$ case (thick blue lines) lies in the middle. Now, if we recall our $m_{n}$ versus $a$ distribution (Figure 1), we note that the two more massive DPs for a fixed total mass belong to the $\alpha=1.8$ distribution. This fact alone tells us that just the two more massive bodies can dominate the heating of the debris disk, despite the fact that most of the less massive bodies of the same $\alpha=1.8$ distribution are less massive than the corresponding objects in the $\alpha=2.2$ distribution (i.e., the presence of larger-mass perturbers excites larger eccentricities and inclinations in the disk, regardless of the presence of lower-mass perturbers.).

Another general feature from Figure 2, for the simulations which do not include the giant planet, is the initial bump evident in both $\langle e\rangle$ and $\langle i\rangle$. This bump results from the initial shock given by DPs to the disk, before the particles reach a relaxed state with the DP-generated potential. After such relaxation, the population continues its $\langle e\rangle$ and $\langle i\rangle$ evolution in a slower and steadier pace. This is typical behavior for the evolution of the whole disk in all our simulations.

It is worth noting that without the giant planet, both the maximum values of $\langle e\rangle$ and $\langle i\rangle$, reached at the top of the initial bump, remain below the maximum $e$ and $i$ of the DP distributions $\left(0^{\circ} .1\right.$ and $5^{\circ}$, respectively). From this, we conclude that the lowest mass explored in this work, approximately the mass of the classical Kuiper Belt, is not enough to excite the average particle eccentricity/inclination up to the excitation level of the DPs, not at the bump nor at any other point of the 1 Gyr simulation (we estimate they would require $10 \mathrm{Gyr}$ or more to reach this level of excitation).

An important result stemming from Figure 2 is the damping effect that a Neptune-like giant planet produces on the stirring of the inclinations and eccentricities of the disk particles, which otherwise would be increased by the perturbations of DPs alone. Indeed, the values reached by $\langle e\rangle$ and $\langle i\rangle$ at the end of the simulation, owing just to the DPs' influence, are $\langle e\rangle \sim 0.07$ and $\langle i\rangle \sim 3{ }^{\circ} .3$, while, when the giant planet is included, the maximum values reached are $\langle e\rangle \sim 0.045$ and $\langle i\rangle \sim 1$.8, for the three different dMD indexes. This effect was previously pointed out by Muñoz-Gutiérrez et al. (2015), noting that an interior giant planet of small eccentricity seems to stabilize the orbits of debris disk particles, preventing the important stirring produced by DPs. Later on, we will discuss the origin of this important phenomenon. However, from this first experiment we can see the weak dependence of such effect on the dMD of DPs: for the three indexes, the damping is of the same order, and the relative behavior, between the different distributions of DPs, is maintained in both $\langle e\rangle$ and $\langle i\rangle$.

\subsection{Effect of Progressively Hotter DPs Populations}

Figure 3 shows the evolution of the global disk quantities $\langle e\rangle$ and $\langle i\rangle$, as well as their standard deviations $\sigma_{e}$ and $\sigma_{i}$; this time the results stand for simulations that include DP populations of 

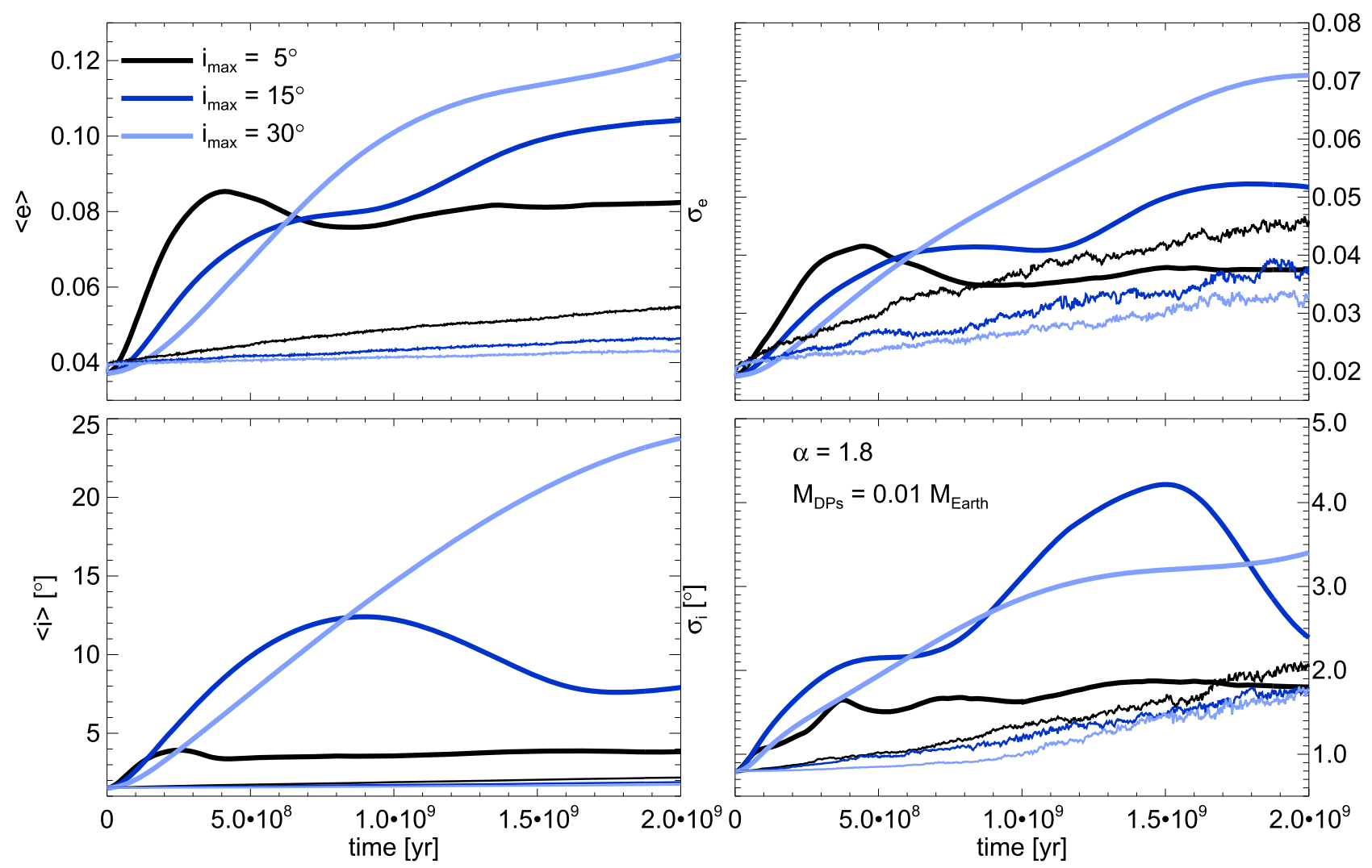

Figure 3. Evolution of the $\langle e\rangle, \sigma_{e},\langle i\rangle$, and $\sigma_{i}$ of the particles of debris disks with $M_{\mathrm{DPs}}=0.01 M_{\oplus}$ and $\alpha=1.8$. Black lines represent cold disks with $i_{\max }=5^{\circ}$, dark blue lines warm disks with $i_{\max }=15^{\circ}$, and light blue lines hot disks with $i_{\max }=30^{\circ}$; same as in the previous figure, thick and thin lines are for simulations without and with the giant planet, respectively.

different vertical excitations. We label the populations as a function of their maximum initial inclination-cold distributions (in black lines) when $i_{\max }=5^{\circ}$, warm distributions (blue lines) when $i_{\max }=15^{\circ}$, and hot distributions (light blue lines) when $i_{\max }=30^{\circ}$. Again, we differentiate the cases including the giant Neptune-like planet (in thin lines) from those without it (in thick lines). The total mass of all DP distributions in this case is $0.01 M_{\oplus}$ and $\alpha=1.8$ in all simulations shown in this section.

We extend these simulations to 2 Gyr (which is further than the $1 \mathrm{Gyr}$ used in the previous section) to better see the evolution of the initial bump produced by the DPs and the subsequent relaxation and steady evolution of the debris disk particles. This relaxation is evident in the thick black curves of Figure 3; one can imagine that the thick dark blue curves are near relaxation, while for the other four simulations it is evident that much more time is needed to reach relaxation.

In Muñoz-Gutiérrez et al. (2015), we had speculated about the maximum average inclination reached by the disk particles in our simulations as a function of the maximum initial inclination of the DP distributions. In Figure 3, we see that this could indeed be the case when the disk evolves without the presence of the giant planet (thick lines), i.e., larger initial DP inclinations imply greater vertical heating of the disk. Nevertheless, some subtleties should be noted: first, the maximum $\langle i\rangle$ reached at the top of the initial bumps in all cases is always below the $i_{\max }$ of the corresponding distribution. Even if the top of the bump is not reached in 2 Gyr for the hot distribution of DPs, its curvature already shows the maximum will likely be below $30^{\circ}$ (maximum of the distribution). On the other hand, the warm and hot distributions can increase the maximum $\langle e\rangle$ of the test particles beyond the maximum $e$ of the DPs (set in all cases to 0.1 ); this is not the case for the cold distribution where $\langle e\rangle$ settles near 0.08 .

Second, the slopes of the black, blue, and light blue thick curves in the left and right panels of Figure 3 are progressively less steep, which means that as the DP inclinations become larger, their effect will be slower but, in the long run, it will be greater. This is evident in $\langle i\rangle$ and $\sigma_{i}$ (Figure 3, bottom panels), but also clear in $\langle e\rangle$ and $\sigma_{e}$ (Figure 3, upper panels). With time, in distributions without the giant planet, the scattering produced by hotter DP distributions on a cold disk will be larger, but the timescale required for this will also be larger. The latter behavior is to be expected as the relative velocities of individual interactions between highly inclined DPs and particles in a cold disk will be greater, and consequently the perturbation experienced by the cometary nuclei will be quicker and therefore smaller (or equivalently, when the DP distribution is hotter, the DPs will spend less time near the disk, which means there will be fewer close encounters when they are hotter). As a confirmation of this point, we note that the previously mentioned initial bump produced by DPs and the subsequent relaxation of the particles are clearly visible in the evolution of $\langle i\rangle$ (bottom-left panel of Figure 3) for the $\left|G P_{N} / \alpha 1.8 / i_{\max } 5 / M_{\mathrm{DP}} 1\right|$ and $\left|G P_{N} / \alpha 1.8 / i_{\max } 15 / M_{\mathrm{DP}} 1\right|$ simulations, but not for the $\left|G P_{N} / \alpha 1.8 / i_{\max } 30 / M_{\mathrm{DP}} 1\right|$, which even after 2 Gyr has not reached the relaxation expected after the initial bump.

The inclusion of the giant planet significantly modifies the behavior. First, as already pointed out, the giant planet damps the scattering produced by the DPs (regardless of their 

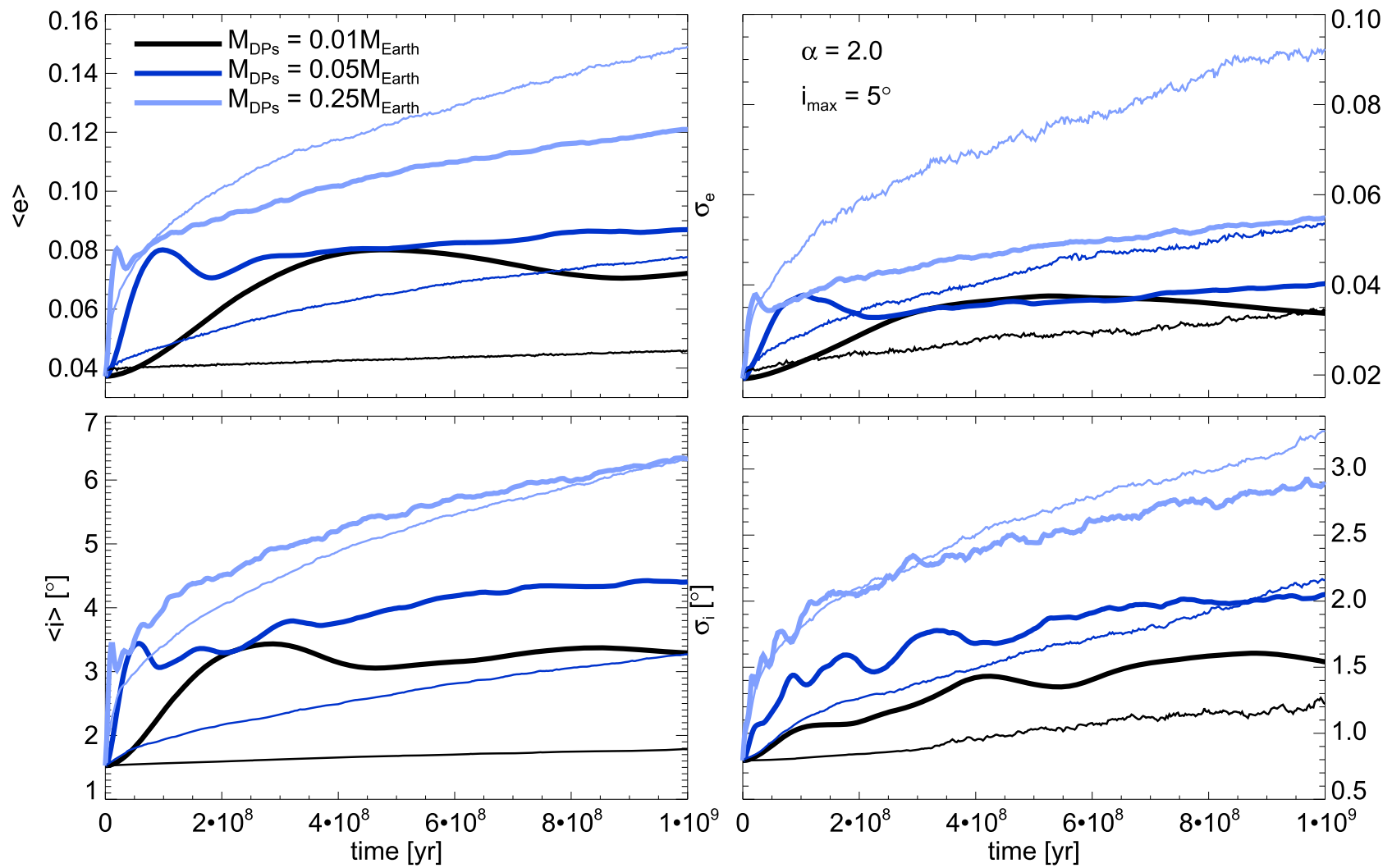

Figure 4. Evolution of the $\langle e\rangle, \sigma_{e},\langle i\rangle$, and $\sigma_{i}$ of the particles of cold debris disks with $i_{\max }=5^{\circ}$ and $\alpha=2.0$. Black lines represent disks with $M_{\mathrm{DPs}}=0.01 M_{\oplus}$, dark blue lines disks with $M_{\mathrm{DPs}}=0.05 M_{\oplus}$, and light blue lines disks with $M_{\mathrm{DPs}}=0.25 M_{\oplus}$; thick lines are for simulations without the giant planet, and thin lines are for simulations including the giant planet.

maximum inclinations), and second, the stirring produced by DPs is weaker for larger inclinations, contrary to the case when only DPs are present. Note the inverse relation between the thin and thick lines at the end of the simulations in all panels of Figure 3. This means that hotter distributions of scatterers will be less effective in heating a cold disk if a stabilizing interior giant planet is present.

From Figure 3, it can be seen that the black curves are initially steeper than the blue ones, but at a certain time they saturate and stop growing earlier than the less steep curves; in the case of $\langle i\rangle$, the expected peak for each distribution is directly related to the $i_{\max }$; therefore, eventually the light blue line should surpass the black one. However, this would take over $100 \mathrm{Gyr}$, much longer than the expected lifetime of the system. In other words, in the presence of the giant planet, the heating produced by hotter DP distributions would require a huge amount of time. For these simulations, it can be said that the effect of the giant only delays the stirring produced by DPs, but for the timescales involved one can safely argue that the giant planet is effectively a stabilizing agent of the debris disk.

\subsection{Effect of Increasing the Total Mass of DPs}

In the simulations shown in Figure 4, we varied the total mass of DP distributions. We present three cases: $0.01 M_{\oplus}$ (black), $0.05 M_{\oplus}$ (blue), and $0.25 M_{\oplus}$ (light blue). In Table 2, these masses correspond to 1, 5, and $25 M_{\mathrm{CKB}}$. As before, simulations that include the giant planet are shown in thin lines while simulations without the giant are shown in thick lines. The constant parameters in these simulations are the maximum inclination of DP distributions, set to $i_{\max }=5^{\circ}$, and the $\mathrm{dMD}$ index, set to $\alpha=2$.

Without the giant planet (thick lines), we observe that as the DPs become more massive, their effect on increasing $e$ and $i$ becomes more rapid (without increasing the initial bump), raising $\langle e\rangle$ and $\langle i\rangle$ to values of $\sim 0^{\circ} .08$ and $\sim 3.5$, respectively, at the top of the initial bump, regardless of the total mass of the scatterers. This implies that the initial rearrangement of the particles will respond to the initial DPs' $e$ and $i$ distributions and not to their mass, but this rearrangement occurs sooner for more massive DPs. After the initial bump, later steady evolution is smooth, both in $\langle e\rangle$ and $\langle i\rangle$. As one would expect, the more massive the DPs, the more scatter they will produce, over a set amount of time, on the disk.

Additionally, simulations $\left|G P_{N} / \alpha 2 / i_{\max } 5 / M_{\mathrm{DP}} 1\right|$ and $\left|G P_{N} / \alpha 2 / i_{\max } 5 / M_{\mathrm{DP}} 5\right|$ seem to reach a saturation limit, i.e., maximum $\langle e\rangle$ and $\langle i\rangle$, after which the DPs can no longer increase the average values of the population. On the contrary, the simulation $\left|G P_{N} / \alpha 2 / i_{\max } 5 / M_{\mathrm{DP}} 25\right|$ shows a monotonically growing $\langle e\rangle$ and $\langle i\rangle$ evolution, even after $\langle i\rangle$ reaches the $i_{\max } \sim 5^{\circ}$ limit that we have previously suggested as the saturation for a distribution of cold DPs. This means that DPs massive enough can stir particle inclinations, at least above their own maximum limit.

Now let us consider the simulations including the giant planet. For the $0.01 M_{\oplus}$ DP distribution, the result from including the giant planet is similar to that seen in the black lines of Figure 3, i.e., the heating suppression due to the giant planet. But, for the most massive DP distribution $\left(0.25 M_{\oplus}\right)$, this behavior reverses, and now, at the end of the simulation, the scattering produced by DPs plus the giant is larger than the 
one produced by DPs alone, as one would expect from adding mass to the scattering population. This behavior is evident in the light blue curves for $\langle e\rangle, \sigma_{e}$, and $\sigma_{i}$ of Figure 4, where the thin lines rapidly surpass the thick ones after just $\sim 50 \mathrm{Myr}$ for the cases of $\langle e\rangle$ and $\sigma_{e}$, and after $\sim 300 \mathrm{Myr}$ for $\sigma_{i}$. Although slower, this also seems to occur for $\langle i\rangle$, near the 1 Gyr limit of our simulations.

This result shows that a stabilizing mass-related threshold exists; this threshold probably depends on the mass of the giant planet (the larger the mass of the giant, the larger the mass of the disk it can stabilize).

For a fixed mass of the giant planet, the threshold depends on the total mass of the disk; below this threshold, a massive interior planet is able to slow down the heating of cometary nuclei due to the action of massive dwarf-planet-sized scatterers, but above such threshold, the giant contributes to the dispersion of the orbits. From Figure 4, we estimate this threshold to be between 0.05 and $0.25 M_{\oplus}$ for the scatterers considering a Neptune-mass giant.

This implies that in the presence of a giant planet, very massive disks will quickly evaporate, but along with the cometary nuclei, many DPs will also disperse until the mass of the disk reaches the threshold value, when the evolution will slow down. We indeed see an increase in the loss of cometary nuclei in the simulations with more massive disks. Besides, when the giant planet is included, at least one DP is lost in every simulation, and even two in the simulation $\left|G P_{Y} / \alpha 1.8 / i_{\max } 15 / M_{\mathrm{DP}} 1\right|$, when their semimajor axes grow larger than $100 \mathrm{au}$.

\subsection{A Mass Threshold for the Stabilizing Effect of the Giant}

The mass threshold we aim to determine depends on the mass of the scatterers (DPs), the mass of the giant planet, as well as its distance to the disk. In the present work, we fixed the mass of the giant planet and its distance to the disk (Neptunelike). Additionally, the giant's eccentricity and inclination could have an important effect; nonetheless, we left the exploration of other parameters for later work. In this paper, we focus on the existence of two regimes: the first one, when the giant planet acts as a stabilizing agent of disk particles, and the second, when the giant also contributes to the dispersion of particles in the disk, once its ordering influence over cometary nuclei is surpassed by DP perturbations.

The two regimes we find can already be guessed from the results shown in Figure 4, mainly those in the top-left panel, where the evolution of $\langle e\rangle$ is plotted for three different cases which differ by the total mass of the DP distributions. In such a figure, there is a noticeable change in the behavior of the $\langle e\rangle$ evolution; when the total mass is $0.05 M_{\oplus}$, the thin dark blue curve remains below the thick dark blue curve, this means that the effect of the giant still results in the stabilization of cometary nuclei. When the mass of DPs is $0.25 M_{\oplus}$, the thin light blue curve rapidly surpasses the thick light blue one, i.e., in such a case, the giant contributes to the heating of the disk. Therefore, the change in regime occurs between 0.05 and $0.25 M_{\oplus}$. To account for the gaps in the intermediate masses and better cover the full mass interval $\left(0.01-0.25 M_{\oplus}\right)$, we added the cases for total masses of 0.0223 and $0.1118 M_{\oplus}$. For this purpose, we ran $1 \mathrm{Gyr}$ integrations of 2000 test particles in the debris disk. We used only cold distributions of DPs for the cases with and without the Neptune-like giant (Table 2 lists the total DP masses covered in this experiment).

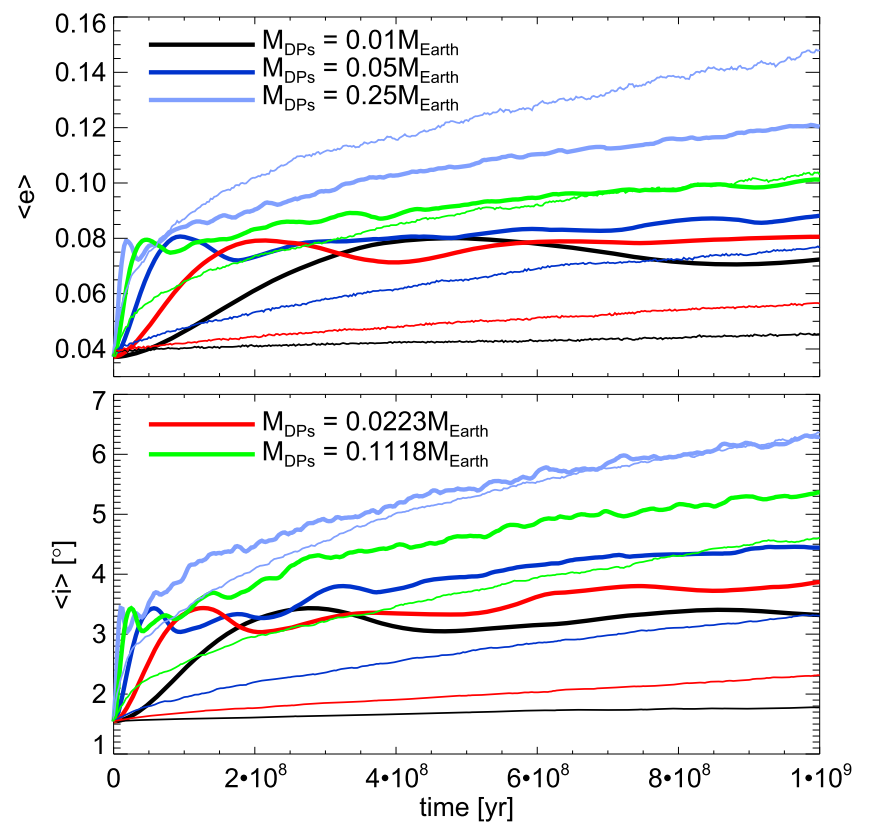

Figure 5. Evolution of the $\langle e\rangle$ and $\langle i\rangle$ of the particles of cold debris disks with $i_{\max }=5^{\circ}$ and $\alpha=2.0$. As in previous figures, black lines represent disks with $M_{\mathrm{DPs}}=0.01 M_{\oplus}$, dark blue lines disks with $M_{\mathrm{DPs}}=0.05 M_{\oplus}$, and light blue lines disks with $M_{\mathrm{DPs}}=0.25 M_{\oplus}$. The two new cases for disks with $M_{\mathrm{DPs}}=0.0223 M_{\oplus}$ and $M_{\mathrm{DPs}}=0.1118 M_{\oplus}$ are shown in red and green lines, respectively. Again, thick lines are for simulations without the giant planet, thin lines for simulations including the giant.

Figure 5 shows the evolution of $\langle e\rangle$ and $\langle i\rangle$ for the five DP distributions, with and without the giant planet. We plotted again as black, dark blue, and light blue curves the evolution of $\langle e\rangle$ and $\langle i\rangle$ for the $0.01,0.05$, and $0.25 M_{\oplus}$ cases, respectively (those shown in Figure 4), but now including only 2000 cometary nuclei in the disk. Reducing the number of test particles from 5000 to 2000 has not modified significantly the statistical evolution of the disk as a whole, as can be seen by comparing the left panels of Figure 4 with the black and blue curves, both thick and thin, of Figure 5 . The new cases $(0.0223$ and $0.1118 M_{\oplus}$ ) are plotted in different colors to highlight the completeness they provided in covering the full mass interval. In red and green, we plotted the evolution of $\langle e\rangle$ and $\langle i\rangle$ for the 0.0223 and $0.1118 M_{\oplus}$ cases, respectively.

The anticipated behavior of both $\langle e\rangle$ and $\langle i\rangle$ is observed for the cases plotted in red and green. In red, the thick and thin lines (with and without the giant) remain between the black and dark blue curves, while the green lines remain between the dark blue and light blue lines.

To determine the mass of the disk when the final $\langle e\rangle$ is the same with or without the giant planet, we plotted in the top panel of Figure 6 the values of the final $\langle e\rangle$ versus the total mass in DPs for the cases when the giant is present in the simulations (blue crosses) and for cases when it is not included (red crosses). Both red and blue crosses show a progressive increment, as expected if a population of scatterers is progressively more massive, i.e., more massive DPs will produce a larger dispersion. For total DP masses below $0.096 M_{\oplus}$, the giant will contribute to maintain a more ordered disk than if only DPs were present, while above such a limiting mass, the giant substantially contributes to the radial heating of the disk. This estimate comes from fitting parabolas (shown as red and blue lines). 


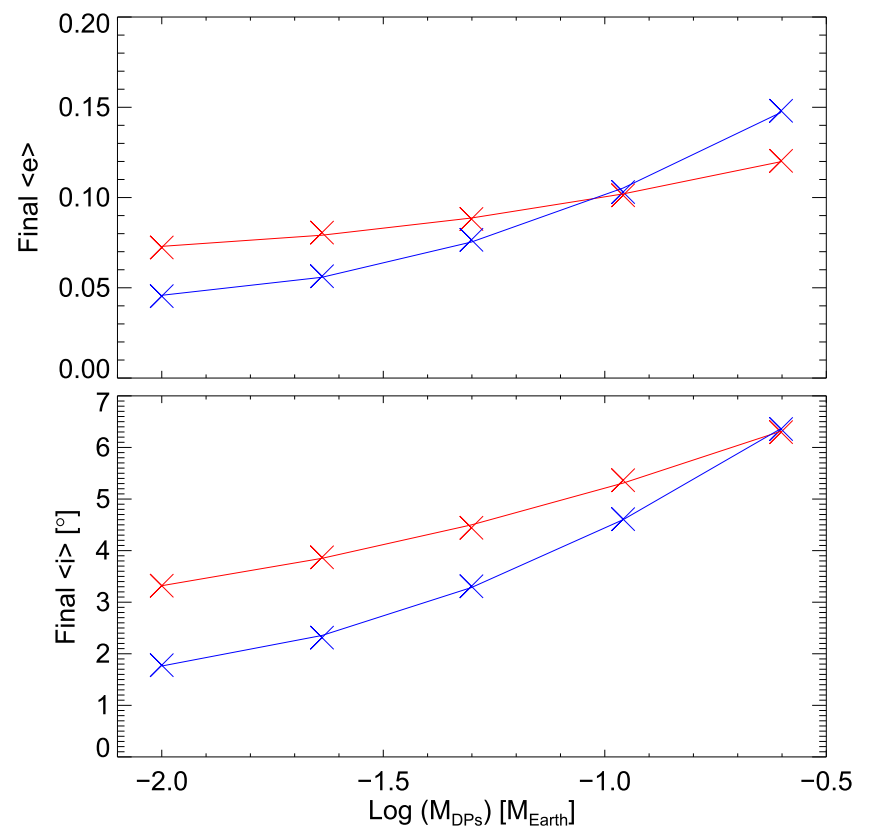

Figure 6. Final values of $\langle e\rangle$ and $\langle i\rangle$ for the different masses of DP distributions used. Red crosses stand for simulations without the giant planet, while blue crosses are for simulations that included the Neptune-like giant. Second-order fittings are used to find the mass where the giant changes regimes, from stabilizing to dispersing particles in the disk.

The bottom panel of Figure 6 shows a similar analysis for $\langle i\rangle$. In this case, the regime changes at $0.238 M_{\oplus}$.

One would expect the efficiency of the heating to be related to the mass of the DPs, and the behavior shown in Figure 6 demonstrates that the dependence in mass is different for both scenarios (with and without the giant planet). In our simulations without the giant planet, we find that the efficiency of the heating is proportional to the mass, as expected; in Figure 7, we plot again the evolution of $\langle e\rangle$ and $\langle i\rangle$ (for the same simulations used in Figure 5), but instead of plotting them directly versus time, we multiply time by the mass of the DPs. There we can see that differences are minimal; on the other hand, when the giant planet is included, the behavior changes dramatically. When studying the dependence of the efficiency of the heating with the mass, we find that the evolution of $\langle i\rangle$ is proportional to the mass of the DPs squared. In Figure 8, we plot the evolution of $\langle e\rangle$ and $\langle i\rangle$, in this case versus time multiplied by the mass of DPs squared. We again see how the $\langle i\rangle$ curves overlap quite nicely; although the overlap of the $\langle e\rangle$ curves is not as good, its behavior is still much better represented with the mass squared than with a linear dependence.

In summary, when no giant planet is present, the rate of heating is proportional to the mass of the DPs; however, when a giant planet is present there is an additional efficiency factor and the heating rate is proportional to the mass squared.

\section{Discussion and Conclusions}

In this work we have explored the evolution of a debris disk that resembles the cold component of the classical Kuiper Belt, but that represents a generic disk in the sense that it is randomly distributed and formed by small-sized particles. Such disk evolves under the gravitational influence of 50 DPs, which represent the 50 heaviest bodies of three different mass

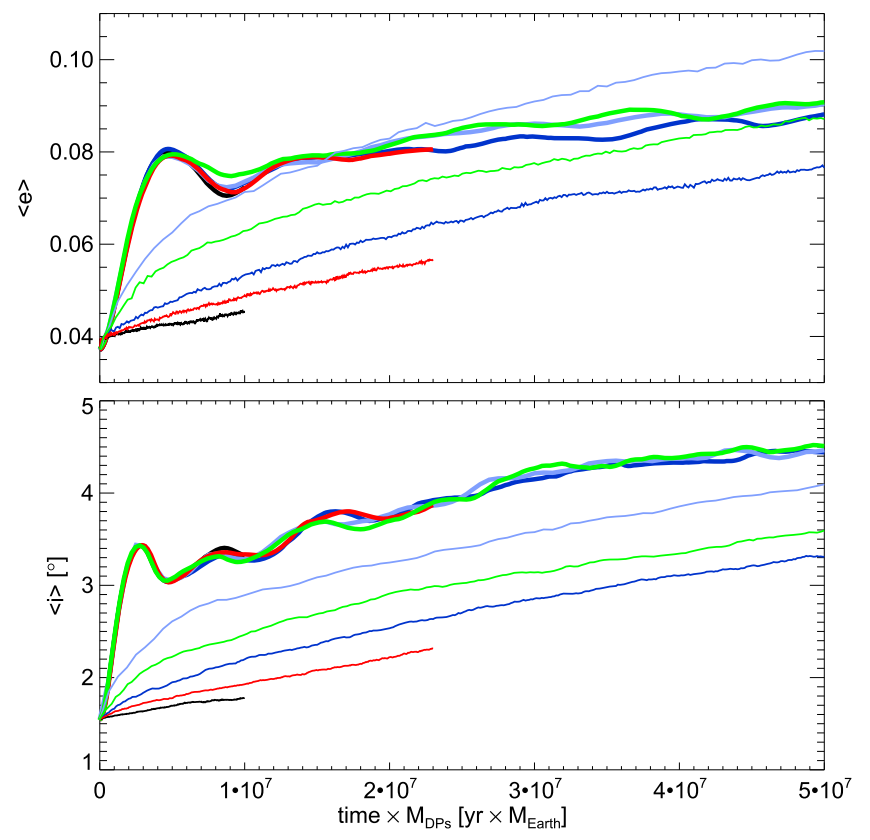

Figure 7. Same as in Figure 5 but instead of time, we plotted $\langle e\rangle$ and $\langle i\rangle$ vs. time multiplied by $M_{\text {DPs. }}$. Thick and thin lines stand for simulations not including and including the giant planet, respectively.

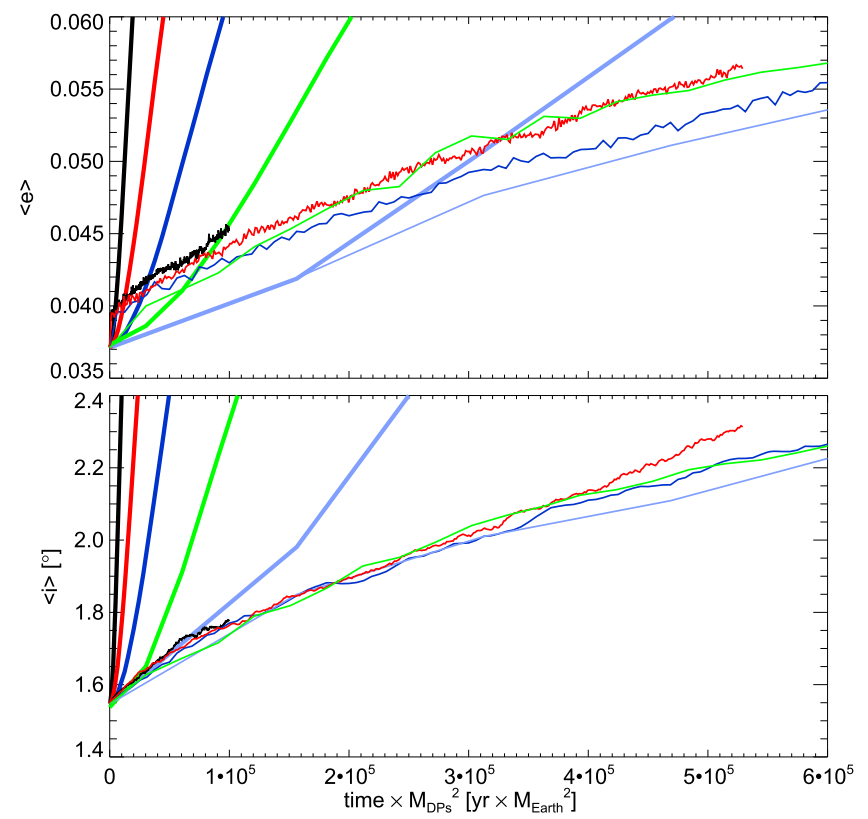

Figure 8. Same as in Figure 5 but we plotted $\langle e\rangle$ and $\langle i\rangle$ vs. time multiplied by $M_{\mathrm{DPs}}^{2}$. Again, thick lines are for simulations without the giant, and thin lines for simulations including the giant planet.

distributions, with and without the presence of a giant planet, similar to Neptune.

Our results have shown that DPs alone induce a progressive stirring in the eccentricities and inclinations of cometary nuclei, proportional to the mass of the scatterers. Such results may be of importance for very external debris disks, i.e., disks that lie away from the influence of any giant planetary body, or for very early protoplanetary disks when such giant bodies have not yet formed (since it is to be expected that at least some DP-sized objects could form in a short time period, during the early evolution of protoplanetary systems). 
On the other hand, when a giant planet is included, we find that the heating rate of the disk is proportional to the square of the mass of the DPs. This produces two regimes for the stirring of particles in the disk. In the first one, when the disk mass is below a certain threshold, the giant planet acts as a stabilizing agent, preventing excessive dynamical heating of cometary nuclei. Above such a threshold, the giant contributes to the scattering of particles. Some consequences can be foreseen from these two regimes on the stirring and dust formation: the presence of a giant planet induces stirring for more massive disks, and restrains stirring for light disks (like the Kuiper Belt).

This tendency would lead us to think that massive debris disks, with an interior giant planet, would produce dust more efficiently in the beginning because of the severe increment on the stirring; this is not so straightforward, since a giant planet would vertically heat the disk, rapidly incrementing its volume, reducing the density, and, with it, reducing the probability of collisions among cometary nuclei. Whether the dilution of the disk or the increment of the stirring will be more important is beyond the scope of this paper. On the other hand, a giant planet with a lighter disk could help sustain it to occupy a given volume for longer timescales while being stirred by DPs; this could provide a more sustained mechanism able to produce some dust in a debris disk for longer timescales. A study on the production of dust with and without the presence of dwarfs and giant planets is needed (and is out of the scope of this paper).

For the current solar system, the combination of Neptune and the mass of the Kuiper Belt implies that Neptune acts as a stabilizer agent for the orbits of the cometary nuclei. Even if a larger number of DPs exist in the Kuiper Belt (besides the four currently classified as such by the IAU), it is unlikely that the mass of the Kuiper Belt would be an order of magnitude larger than is currently estimated; therefore, it is hard for them to significantly contribute to the dispersion of the objects that form the hot classical belt or the scattered disk. Nonetheless, a detailed study is still necessary to account for the contribution, in the heating of primordial objects in the cold classical belt, from known and from yet to be discovered DPs in the Kuiper Belt; this could lead to the explanation for the smooth transition between the cold and hot classical populations of the Kuiper Belt.

We acknowledge an anonymous referee for a careful and detailed report that helped to improve the present work. We acknowledge grant CONACyT Ciencia Básica 255167. M.A.M. and B.P. acknowledge grant DGAPA-PAPIIT IN114114. A.P. acknowledges grant DGAPA-PAPIIT IN109716. We acknowledge the use of the Atocatl supercomputer at the Instituto de Astronomía of the Universidad Nacional Autónoma de México, where most of the simulations presented in this work were performed.

\section{References}

Aumann, H. H., Beichman, C. A., Gillett, F. C., et al. 1984, ApJL, 278, L23 Backman, D. E., \& Paresce, F. 1993, in Protostars and Planets III (Baltimore, MD: STScI), 1253

Bernstein, G. M., Trilling, D. E., Allen, R. L., et al. 2004, AJ, 128, 1364

Chambers, J. E. 1999, MNRAS, 304, 793

Chiang, E., Lithwick, Y., Murray-Clay, R., et al. 2007, in Protostars and Planets V, ed. B. Reipurth, D. Jewitt, \& K. Keil (Tucson, AZ: Univ. Arizona Press), 895

Cotten, T. H., \& Song, I. 2016, ApJS, 225, 15

Dohnanyi, J. S. 1969, JGR, 74, 2531

Fraser, W. C., Brown, M. E., Morbidelli, A., Parker, A., \& Batygin, K. 2014, ApJ, 782, 100

Fraser, W. C., \& Kavelaars, J. J. 2008, Icar, 198, 452

Fraser, W. C., Kavelaars, J. J., Holman, M. J., et al. 2008, Icar, 195, 827

Haisch, K. E., Jr., Lada, E. A., \& Lada, C. J. 2001, ApJL, 553, L153

Kenyon, S. J., \& Bromley, B. C. 2004, AJ, 127, 513

Kral, Q., Clarke, C., \& Wyatt, M. 2017, arXiv:1703.08560

Lee, E. J., \& Chiang, E. 2016, ApJ, 827, 125

Mannings, V., \& Barlow, M. J. 1998, ApJ, 497, 330

Matthews, B. C., Krivov, A. V., Wyatt, M. C., Bryden, G., \& Eiroa, C. 2014, in Protostars and Planets VI, ed. H. Beuther et al. (Tucson, AZ: Univ. Arizona Press), 521

Morbidelli, A., Levison, H. F., \& Gomes, R. 2008, in The Solar System Beyond Neptune, ed. M. A. Barucci et al. (Tucson, AZ: Univ. Arizona Press), 275

Moro-Martín, A., Wyatt, M. C., Malhotra, R., \& Trilling, D. E. 2008, in The Solar System Beyond Neptune, ed. M. A. Barucci et al. (Tucson, AZ: Univ. Arizona Press), 465

Mouillet, D., Larwood, J. D., Papaloizou, J. C. B., \& Lagrange, A. M. 1997, MNRAS, 292, 896

Muñoz-Gutiérrez, M. A., Pichardo, B., Reyes-Ruiz, M., \& Peimbert, A. 2015, ApJL, 811, L21

Mustill, A. J., \& Wyatt, M. C. 2009, MNRAS, 399, 1403

Nesvold, E. R., \& Kuchner, M. J. 2015, ApJ, 798, 83

Nesvold, E. R., Naoz, S., \& Fitzgerald, M. 2017, ApJL, 837, L6

Nesvold, E. R., Naoz, S., Vican, L., \& Farr, W. M. 2016, ApJ, 826, 19

Nesvorný, D. 2015, AJ, 150, 73

Nesvorný, D., Jenniskens, P., Levison, H. F., et al. 2010, ApJ, 713, 816

Oudmaijer, R. D., van der Veen, W. E. C. J., Waters, L. B. F. M., et al. 1992, A\&AS, 96, 625

Smith, B. A., \& Terrile, R. J. 1984, Sci, 226, 1421

Vitense, C., Krivov, A. V., Kobayashi, H., \& Löhne, T. 2012, A\&A, 540, A30 Wyatt, M. C. 2008, ARA\&A, 46, 339

Wyatt, M. C., \& Dent, W. R. F. 2002, MNRAS, 334, 589

Wyatt, M. C., Dermott, S. F., Telesco, C. M., et al. 1999, ApJ, 527, 918 